\title{
Software en línea para el aprendizaje conceptual de la óptica física y geométrica
}

\section{Online software for the conceptual learning of physical and geometric optics}

\author{
Ronald Omar Estela Urbina, ${ }^{1 a}$ Elisa Contreras Barsallo, ${ }^{2}$ Yajaira Lizeth Carrasco Vega, ${ }^{3}$ \\ Benjamin David Carril Verastegui, ${ }^{4}$ Daniel Jesús Castro Vargas, ${ }^{5}$ \\ Jonathan Abrahan Sueros Zarate ${ }^{6}$
}

\author{
Universidad Nacional Intercultural "Fabiola Salazar Leguía" de Bagua ${ }^{13}$ \\ I. E. Túpac Amaru de Chiriaco-Amazonas ${ }^{2}$ \\ Universidad Nacional de Trujillo ${ }^{4}$ \\ Universidad Nacional Autónoma de Chota ${ }^{5}$ \\ Universidad de Ciencias Aplicadas ${ }^{6}$
}

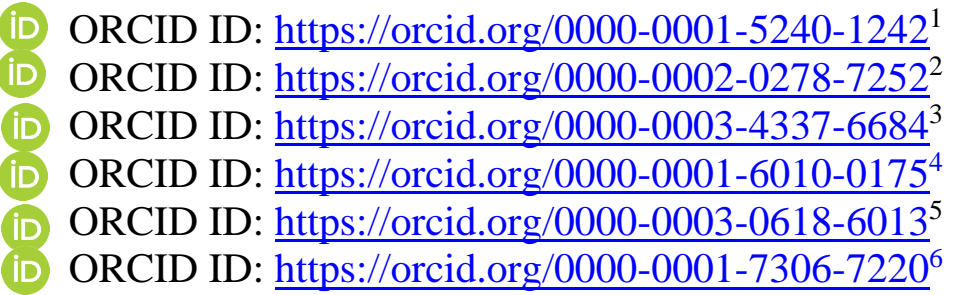

Recibido: 01 mayo de 2021

Aceptado: 03 de setiembre de 2021

\section{Resumen}

El objetivo de la presente investigación fue determinar el nivel de ganancia del aprendizaje conceptual utilizando simuladores en línea en el desarrollo del curso de Física III de la carrera profesional de Ingeniería Civil en los estudiantes de la Universidad Nacional Intercultural "Fabiola Salazar Leguía" de Bagua (Perú), por medio del factor o ganancia normalizada de Hake. La metodología que se llevó a cabo es de enfoque cuantitativo y diseño cuasi experimental, y consistió en recolectar información antes (pre) y posterior a la utilización de simuladores y software en línea (post) en sesiones de clase virtual con los estudiantes matriculados en el mencionado curso. Como resultado logramos determinar una efectividad media de esta metodología, representada por un valor de 0.65 en la temática de óptica geométrica y 0.60 en la óptica física de ganancia normalizada de Hake.

Palabras claves: óptica, software en línea, aprendizaje conceptual, simulación.

\section{Abstract}

The objective of this research was to determine the level of gain in conceptual learning using online simulators in the development of the Physics III course of the Civil Engineering 
professional career in students of the National Intercultural University "Fabiola Salazar Leguía" of Bagua (Peru), by means of Hake's normalized factor or gain. The methodology that was carried out is of quantitative approach and quasi-experimental design, it consisted of collecting information before (pre-test) and after the use of simulators and online software (post-test) in virtual class sessions with students enrolled in the aforementioned course. As a result, we were able to determine an average effectiveness of this methodology, represented by a value of 0.65 in the geometric optics theme and 0.60 in Hake's normalized gain physical optics.

Keywords: optics, online software, conceptual learning, simulation.

\section{Introducción}

Las condiciones actuales de restricciones sociales obligatorias que se vive debido a la emergencia sanitaria mundial y nacional en el Perú, hace propicia la oportunidad para una reinvención docente, un replanteamiento de la metodología tradicional, aunque esto implique un mayor compromiso y dedicación del docente, así como la participación activa del estudiante en el proceso de su propio aprendizaje (Cifuentes, 2020).

Asimismo, la puesta en marcha de la educación virtual universitaria ha representado un cambio "brusco a la forma "habitual" de impartir clases en la modalidad presencial (Delgado et al., 2021), no solo en la temporalidad de la programación de las sesiones, sino también en la adecuación de estrategias y los materiales educativos. En este contexto ha quedado en evidencia las dificultades de esta adecuación virtual, así como la poca preparación docente en tecnología de información (Gutiérrez \& Castillo, 2020).

En efecto, las tecnologías de la información (TIC) y la disponibilidad de software libre en la web constituyen un apoyo en las sesiones de clase(Rodríguez et al., 2019), de tal forma que invitan al usuario a un aprendizaje autónomo, y se debería pensar en que estas "herramientas" o "recursos" deben ser incorporadas también a una planificación curricular permanente sea presencial o virtual la modalidad de estudio (Solé et al., 2020). En este sentido, la física es una ciencia experimental que precisa complementar la parte teórica con recreaciones o simulaciones de un hecho físico (Zenteno et al., 2020), para lo cual la tecnología de la información juega un papel muy importante como soporte en este contexto (Estela et al., 2021). En el caso especial de la física universitaria, no solo se dispone de simuladores de la temáticas referidas a la mecánica clásica, sino también a mecánica relativista y física moderna (Siswoyo \& Muliyati, 2021). 


\section{Revisión de literatura}

\section{Currículo universitario de ingenierías y la óptica}

Las competencias específicas que se pretenden desarrollar en los estudiantes y futuros egresados de las carreras profesionales de ingeniería se circunscribe en muchos casos a aplicaciones prácticas del conocimiento físico, temáticas como: mecánica, calor o electricidad; sin embargo, los contenidos de física moderna, óptica o mecánica cuántica (Solbes et al., 2019) no se abordan, debido a la exigencia del equipamiento de laboratorio especializado, que en numerosos casos no se cuenta, o también debido a que los tópicos del conocimiento científico sean poco utilizados en su quehacer profesional (Estela \& Contreras, 2020). Esta realidad no solo se evidencia en el nivel universitario, sino también en la Educación Básica Regular (EBR) como lo indica la figura 1 (Jimenez et al., 2020).

Por otro lado, es cierto que un gran número de estudiantes no cuenta con las habilidades para diferenciar la mecánica clásica y la cuántica; así como hay poco dominio respecto a categorizar partículas atómica y subatómicas (Gonzáles et al., 2020). Una de las razones que se puede esbozar a esta realidad es que estos conceptos y fenómenos requieran un tanto de “abstracción”(Merino et al., 2019). Por ello, resulta importante la labor docente para desarrollar una física conceptual, más allá de restringirse a la solución de ejercicios, que en su mayoría convierten al estudiante en un ser mecánico con escasa reflexión y criticidad respecto al mundo físico circundante (Culzoni et al., 2020).

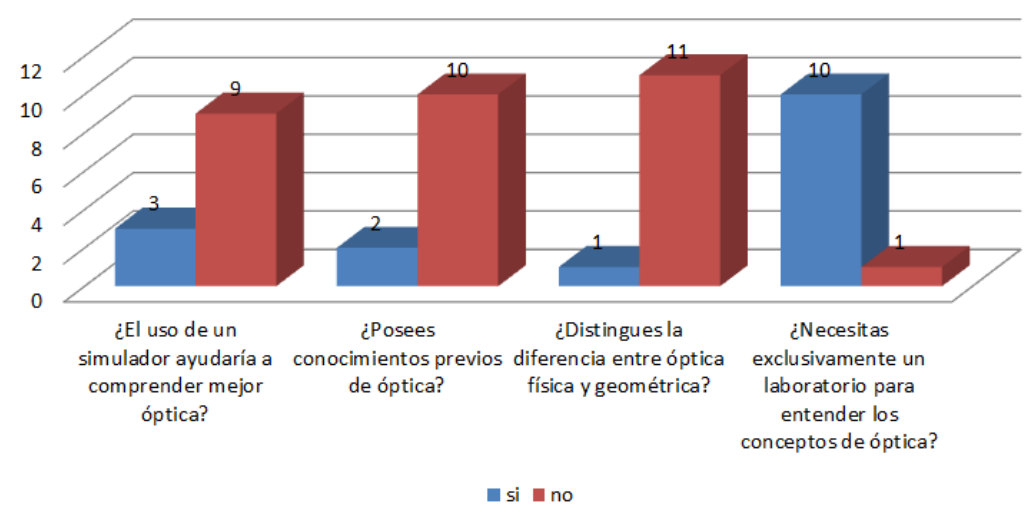

Figura 1. Encuesta diagnóstica

Fuente: Elaboración propia

\section{La importancia de las ideas previas en la clase de óptica}

Una de las formas para potencializar de manera adecuada el performance de nuestra clase: conocimiento de los saberes previos de los estudiantes (Perdomo \& Galo, 2020), sus 
necesidades y expectativas respecto a los alcances del curso (Cruz, 2020). La modalidad de estudios universitarios de aprendizaje no presencial cuenta con dos momentos: asíncrono (aprendizaje autónomo) y síncrono (encuentro virtual por medio de una videoconferencia).

Esta forma de trabajo coloca al estudiante en una posición de protagonista (Barragán, 2020). Esto significa que el docente proporciona material, así como programa actividades que permiten que el estudiante de manera individual, y en algunas veces en forma colaborativa construya su conocimiento (Martínez \& Riveros, 2019), de tal forma que la clase en videoconferencia (el tiempo de encuentro sincrónico con el docente) no requiere periodos prolongados de explicación temática, todo lo contrario permite que sea un espacio para la puesta en marcha de estrategias significativas (Jimenez et al., 2020).

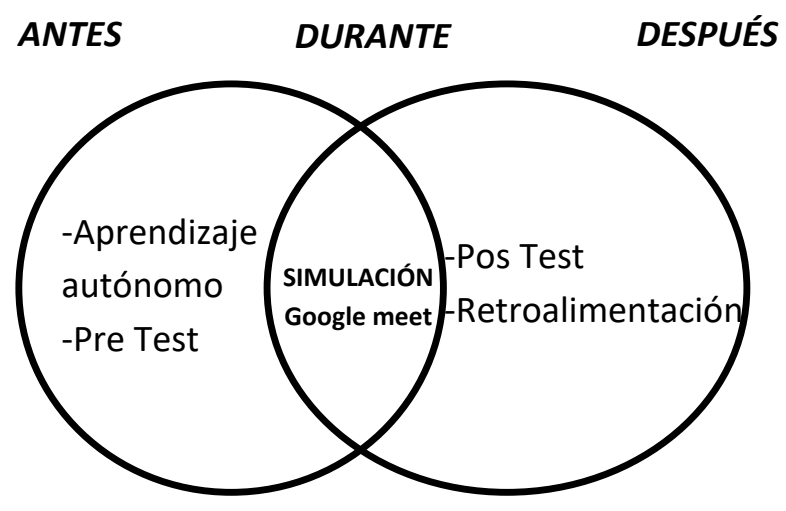

Figura 2. Aprendizaje síncrono y asíncrono Fuente: Elaboración propia

\section{Software en línea para óptica}

Para desarrollar competencias en los estudiantes acordes con las carreras profesionales en las condiciones de educación no presencial, se requiere echar mano de herramientas digitales (Barragán, 2020), que permitan ser en cierta forma un efecto reemplazante del trabajo de laboratorio presencial, sobre todo para aquellas asignaturas cuyo componente práctico es considerable, esto conlleva a que el docente y estudiante explore en el basto mundo del internet aplicaciones y software que apoyen la labor de enseñanza-aprendizaje (Cruz, 2020). El uso de un programa informático en línea no sustituye al docente, todo lo contrario conlleva a una planificación pertinente de cómo aplicar esa estrategia de manera apropiada generando un entorno lúdico, de entrenamiento y aprendizaje conceptual (Miranda \& Romero, 2019). 


\section{Tabla 1}

Simuladores en línea para óptica física y geométrica

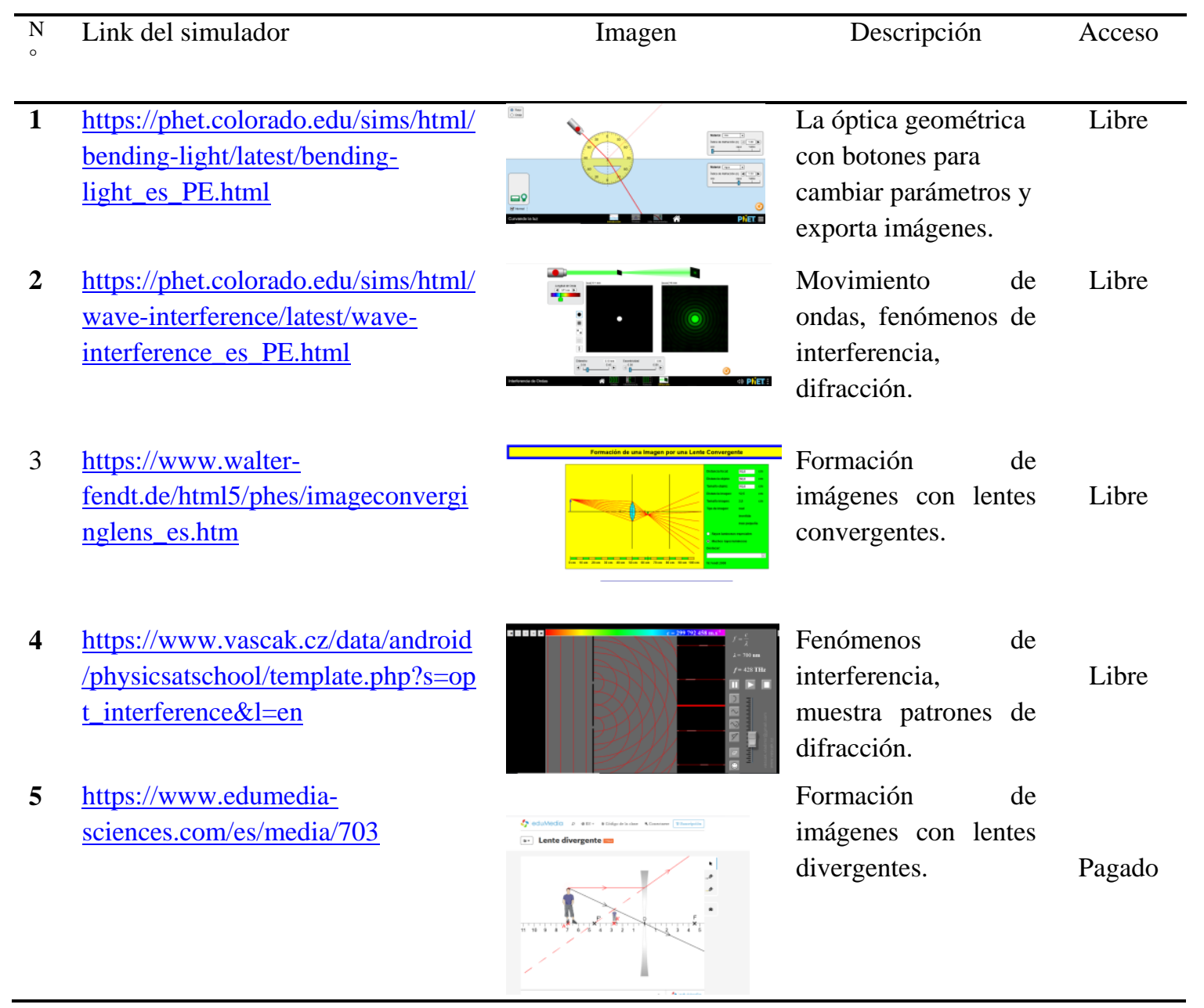

\section{Del experimento en laboratorio a la simulación de la óptica}

La física es una ciencia experimental por excelencia y la óptica es uno de los pilares de esta, lo cual indica que el uso del laboratorio resulta fundamental como complemento teórico de la asignatura, y así lograr la consolidación de las competencias trazadas en el plan curricular (Castiblanco et al., 2019). En el trabajo práctico de laboratorio el aprendizaje del estudiante se evidencia reproduciendo la realidad, trabajando en equipo, siguiendo el método científico como es: la observación, hipótesis, registro de datos y sus respectivas conclusiones (Martínez, Guilcapi, López, \& Mata, 2020). Mientras que el laboratorio en línea pretende recrear de forma virtual todas estas condiciones físicas controladas resultando mucho más eficiente, en cuanto a la reproducción de los fenómenos, y el número de veces de cálculos matemáticos que se pueden realizar con más facilidad (Ribeiro et al., 2019). En estas condiciones se logra la comprensión de nuevas categorías conceptuales de parte de los estudiantes, al conocer de manera lúdica la 
temática de óptica geométrica y física, así como su experimentación virtual (Martínez \& Riveros, 2019).

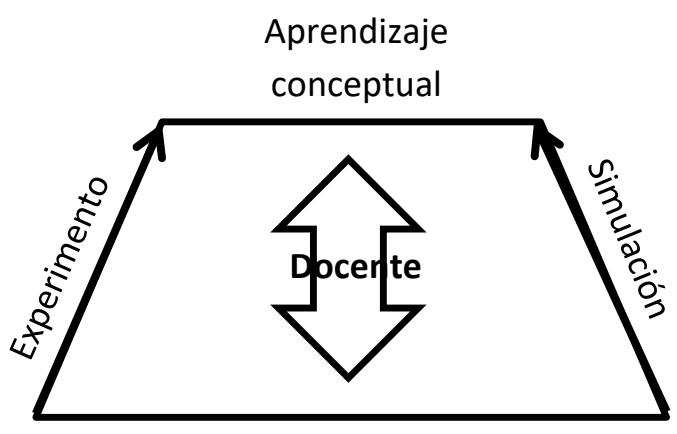

Fundamento teórico de Óptica

Figura 3. Trapecio metodológico en la enseñanza de la óptica Fuente: Elaboración propia

A la luz de todos estos antecedentes, el objetivo de la presente investigación fue determinar el nivel de ganancia del aprendizaje conceptual por medio del facto de Hake, de la temática de óptica utilizando simuladores en línea. Se desarrolló en el curso de Física III, de la carrera profesional de Ingeniería Civil, en los estudiantes de la Universidad Nacional Intercultural "Fabiola Salazar Leguía" de Bagua.

\section{Metodología}

Esta investigación desarrolló un enfoque cuantitativo con diseño cuasi experimental, y se determinó el nivel de ganancia del aprendizaje conceptual de la física óptica y geométrica (Torres et al., 2020), luego de utilizar software o simuladores en línea en las sesiones de clase (Perdomo \& Galo, 2020). Esta metodología se desarrolló con los 12 estudiantes(Cabrejos et al., 2021) matriculados en el curso de Física III de la carrera profesional de ingeniería civil, que en condiciones de educación no presencial, se buscó que mejoren el dominio conceptual de la temática de óptica (Becerra et al., 2020).

La técnica empleada consistió en recolectar información antes (pre) y posterior a la utilización en sesiones de clase virtual de simuladores y software en línea (post), ponderando de forma independiente los datos obtenidos con la variable que se tiene relación, es decir aprendizaje de conceptos de óptica física y geométrica (Perdomo \& Galo, 2020).

Del mismo modo, la indagación se realizó con el aula de Física III, de la carrera profesional de Ingeniería Civil de la Universidad Nacional Intercultural "Fabiola Salazar Leguía” de Bagua - UNIFSLB, del tercer ciclo de estudios no presenciales o virtuales. Respecto 
a la condición de los cuasi experimentos, la muestra que se tomó en cuenta es no probabilística o dirigida, bajo el método de muestreo intencional o de conveniencia (Perdomo \& Galo, 2020). Al ser nuestra población los estudiantes que cursan Física III, se tomó como muestra los 12 estudiantes (Rondon et al., 2020), matriculados en el mencionado curso de modalidad virtual. Los cuales fueron informados respecto al estudio, y se recolectaron los datos previo consentimiento informado (Cabrejos et al., 2021).

Para el recojo de la información se utilizaron los siguientes instrumentos: 1) Encuesta diagnóstica que se aplicó a la totalidad de estudiantes. Esta consistió en unas 15 preguntas, cuyo objetivo fue conocer la percepción que tienen los estudiantes respecto al uso y pertinencia de un simulador en línea, conocimientos previos de óptica, así como ideas de cómo conciben la educación virtual de la física. 2) Pre test, cuestionario de 10 preguntas sobre dominio conceptual a nivel básico e intermedio de la óptica física y geométrica, así como ejercicios de aplicación de los principios que rigen los fenómenos ópticos. 3) Post test, cuestionario que contenía las mismas preguntas del pre test, el cual se aplicó luego de utilizar el simulador.

Con respecto a la validación y confiabilidad de los instrumentos aplicados, se tomó como base cuestionarios que han sido sometidos a juicio de expertos, pasando por todas las pruebas estadísticas correspondientes. El primer instrumento se adaptó del test titulado "Procrastinación académica entre estudiantes de cursos en línea"(García \& Silva, 2019), el segundo y tercer cuestionario se adecuó del "Test conceptual sobre fenómenos de reflexión" (Medina et al., 2019), así como del "Cuestionario para determinar las representaciones de conceptos y procesos físicos en estudiantes" (Flores et al., 2020).

El nivel de dificultad de las preguntas que conformaron el cuestionario de entrada y salida de evaluación (Baidal et al., 2019) fueron equivalentes a exámenes de Física III de los ciclos anteriores, adaptadas al contexto de educación virtual actual (Culzoni et al., 2021), teniendo en cuenta aquellas interrogantes que permitan evidenciar el dominio conceptual de óptica física y geométrica (Naizaque, 2020). Los ítems constaban de múltiple alternativa y de respuesta única (Castillo et al., 2020).

Por su parte, el factor de la ganancia de Hake representa el desarrollo del aprendizaje de un grupo de estudiantes, de la misma forma con el conocimiento de este factor nos conduce a evaluar la efectividad de la aplicación de una metodología en el proceso de enseñanza aprendizaje. La expresión matemática que define este factor es la división de la diferencia entre el post test y pretest entre el aumento máximo posible(Castillo et al., 2020). 


$$
g=\frac{\left\langle \% \text { Nota }_{\text {pos }}\right\rangle-\left\langle \% N o t a_{\text {pre }}\right\rangle}{100 \%-\left\langle \% \text { Nota }_{\text {pre }}\right\rangle}
$$

Este factor indica que, para un grupo de estudiantes dentro del proceso enseñanza aprendizaje de forma tradicional, la ganancia (g) adopta valores por debajo de 0.3 (Castillo et al., 2020). En cambio, para escenarios donde se aplica metodologías activas donde implica un trabajo individual y colaborativa de los estudiantes; de la misma forma cuando existe interacción entre estudiantes-estudiantes, así como estudiante-docente se obtienen ganancias mayores a 0.3 ver tabla 2 (Martínez \& Riveros, 2019).

\section{Tabla 2}

Niveles de ganancia normalizada de Hake

\begin{tabular}{lc}
\hline Ganancia normalizada & Rango \\
\hline Alta & $0.7<g \leq 1$ \\
Media & $0.3<g \leq 0.7$ \\
Baja & $g \leq 0.3$ \\
\hline
\end{tabular}

\section{Resultados}

Se aplicó el cuestionario de Pre test de manera diferenciada en cuanto a la temática. El primero en evaluarse fue el de óptica geométrica a la totalidad de los estudiantes matriculados. El cuestionario consta de 10 ítems, cuyo valor por pregunta fue de 2 puntos, cuyo máximo valor de calificación es 20. El balance del pre test fue que ninguno de los estudiantes logró aprobar la evaluación, logrando obtener en promedio una nota de 4.67 en la escala vigesimal. Los cuestionarios son evaluaciones en línea a través del aula virtual de la UNIFSLB, teniendo una duración de 30 minutos de un solo intento. 


\section{Tabla 3}

Número de respuestas correctas y nota obtenida en el pre y post test para el tema de óptica geométrica

\begin{tabular}{ccccc}
\hline \multirow{2}{*}{ Estudiante } & \multicolumn{2}{c}{ Pre-Test } & \multicolumn{3}{c}{ Pos-Test } \\
\cline { 2 - 5 } & $\begin{array}{c}\text { Respuestas } \\
\text { correctas }\end{array}$ & Nota & $\begin{array}{c}\text { Respuestas } \\
\text { correctas }\end{array}$ & Nota \\
$\mathbf{1}$ & 2 & 04 & 8 & 16 \\
$\mathbf{2}$ & 4 & 08 & 10 & 20 \\
$\mathbf{3}$ & 2 & 04 & 6 & 12 \\
$\mathbf{4}$ & 1 & 02 & 6 & 12 \\
$\mathbf{5}$ & 0 & 0 & 6 & 12 \\
$\mathbf{6}$ & 3 & 06 & 7 & 14 \\
$\mathbf{7}$ & 1 & 02 & 8 & 16 \\
$\mathbf{8}$ & 2 & 04 & 9 & 18 \\
$\mathbf{9}$ & 3 & 06 & 8 & 16 \\
$\mathbf{1 0}$ & 2 & 04 & 9 & 18 \\
$\mathbf{1 1}$ & 5 & 10 & 9 & 18 \\
$\mathbf{1 2}$ & 3 & 06 & 10 & 20 \\
\hline
\end{tabular}

Fuente: Elaboración propia

En los resultados del pretest de óptica geométrica se puede apreciar que el promedio de respuesta acertada es de 2.3 de 10 puntos, lo cual indica un nivel muy bajo de dominio conceptual, así como manejo de principios físicos fundamentales.

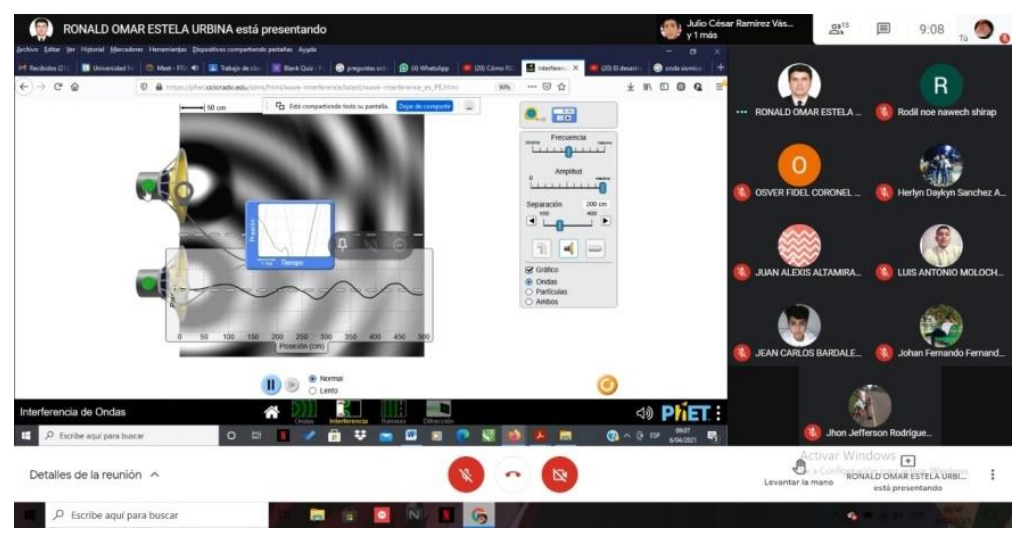

Figura 4. Sesión síncrona utilizando simuladores en línea Fuente: Elaboración propia

Posteriormente a la aplicación del Pre test se explicaron de manera detallada los conceptos de óptica geométrica, así como las leyes que rigen los comportamientos de los fenómenos físicos pertinentes. Toda esta sesión virtual sincrónica de clase se desarrolló aplicando el uso de simuladores en línea, que fue utilizado por el docente a modo de 
explicación. Luego se realizó una demostración personal del uso de los mencionados softwares, quedando en evidencia las intervenciones de sus compañeros con sus aportes para mejorar la comprensión de los fenómenos y la utilización del software.

El cálculo y posterior análisis del factor de ganancia de Hake, se desarrolló tomando en cuenta la tabla 3, de donde se puede extraer el número de respuestas correctas en el pre y post test. Teniendo en cuenta que el número máximo de aciertos es 10. De los datos organizados en la tabla extraemos que el puntaje total de aciertos en el pre y post test son 28 y 88 respectivamente.

$$
\begin{gathered}
\text { Puntaje }_{\text {máximo }}=12 \times 10=120 \\
\text { Puntaje }_{\text {pre-test }}=28 \times 100=2800 \\
\text { Puntaje }_{\text {pos-test }}=88 \times 100=8800 \\
g=\frac{\frac{8800}{120}-\frac{2800}{120}}{100-\frac{2800}{120}}=0.65
\end{gathered}
$$

De los datos obtenidos tanto en el pre y post test se obtiene un factor de Hake de 0.65 dejando en evidencia una mejora y ganancia en el aprendizaje conceptual en la óptica geométrica normalizado por Hake en nivel medio de ganancia.

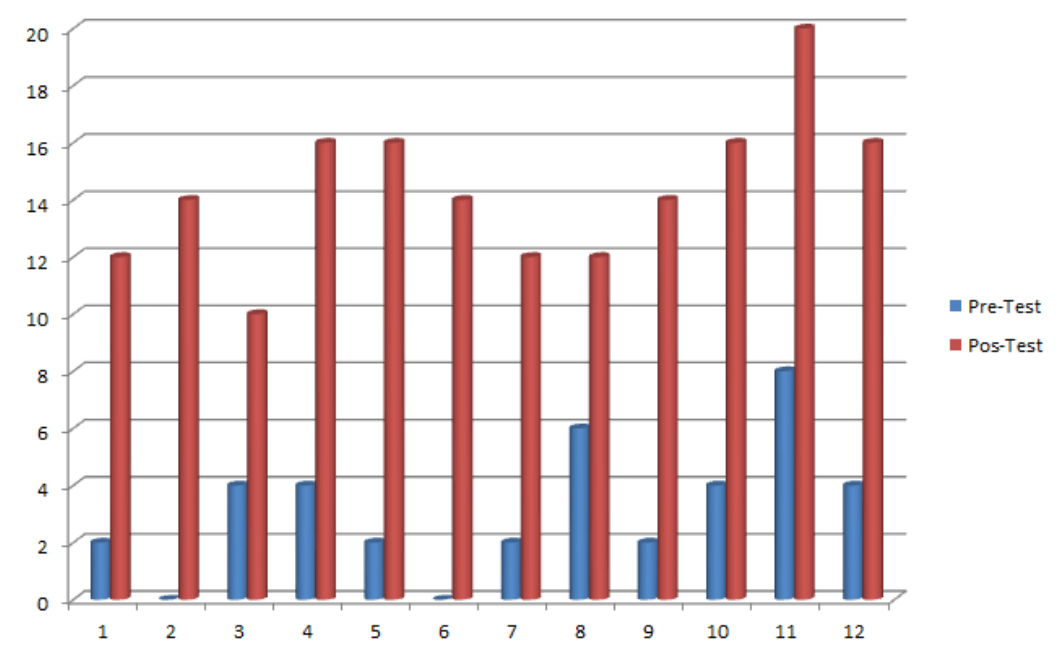

Figura 5. Nota obtenida en el pre y post test para el tema de óptica física - difracción

Fuente: Elaboración propia 
Se realizó la misma metodología para el componente curricular de la óptica física con los mismos estudiantes de la carrera profesional de ingeniería civil, de la misma forma se elaboró un cuestionario con preguntas que abordaban la parte conceptual y aplicaciones de principios fundamentales de la óptica física. Se obtuvo en promedio una nota de pre test de 3.17 y 14.3 de pos test, aplicando la fórmula de Hake. Para esta temática se obtuvo un factor de ganancia de 0.60 .

$$
\begin{gathered}
\text { Puntaje }_{\text {máximo }}=12 \times 10=120 \\
\text { Puntaje }_{\text {pre-test }}=19 \times 100=1900 \\
\text { Puntaje }_{\text {pos-test }}=80 \times 100=8000 \\
g=\frac{\frac{8000}{120}-\frac{1900}{120}}{100-\frac{1900}{120}}=0.60
\end{gathered}
$$

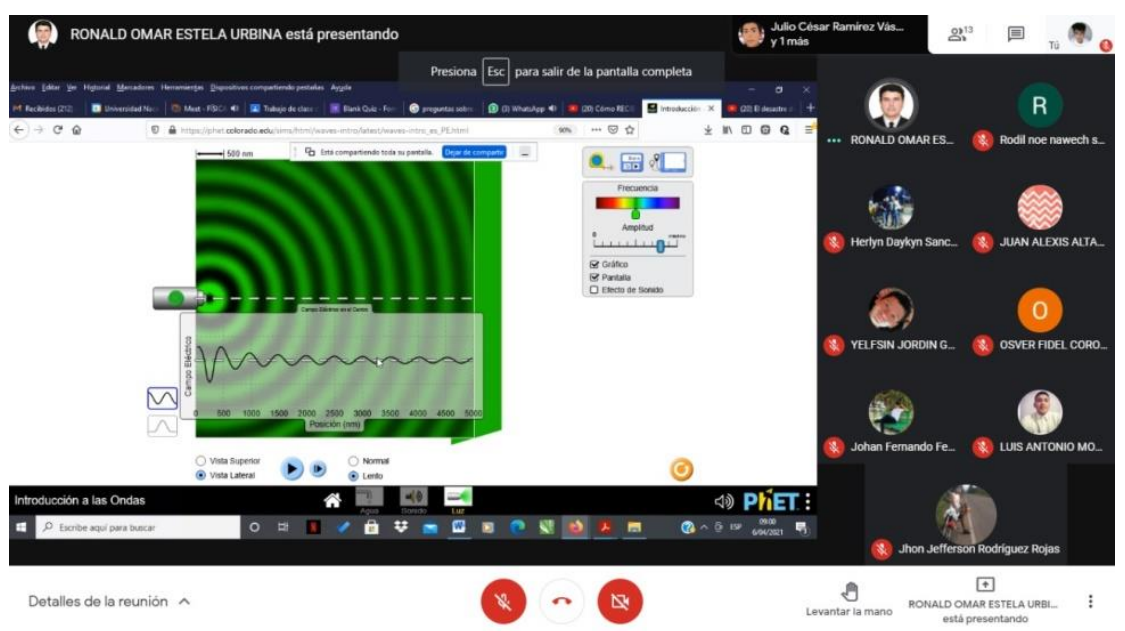

Figura 6. Sesión síncrona utilizando simuladores en línea

Fuente: Elaboración propia

\section{Discusión}

En base a la investigación que se realizó debemos indicar que dentro de la estructura curricular de física no se suele desarrollar la temática referente a óptica física y geométrica, y esta realidad se evidencia desde la educación Básica Regula (EBR) según la evaluación diagnostica realizada. Una de las razones que se puede indicar es la falta de laboratorios especializados o el poco dominio de simuladores en línea, así como su fomento de las tecnologías de información (Culzoni et al., 2020). 
En los entornos de enseñanza virtual, sobre todo en el trabajo asincrónico(donde el docente y estudiante no necesariamente están conectados simultáneamente), se enfatiza el hecho de que el estudiante pueda ser gestor de su propio aprendizaje (Espinoza, 2020), ya que el uso de un software o simulador suele ser "instructivo" o "intuitivos", y se aprende o se familiariza explorando. Sin embargo, el aprendizaje direccionado por un docente resulta de mucha necesidad sobre todo cuando se dispone de finalidades educativas claras, para generar aprendizajes significativos y duraderos (Albiter et al., 2019). No es solo usar el software para "dinamizar" la virtualidad, sino es necesario ver el progreso y avance de los estudiantes respecto a un programa de estudios (Lara et al., 2019). El uso de metodologías activas que impliquen la interacción docente - estudiante pone en segundo plano al docente como el "poseedor" del conocimiento, todo lo contrario empodera al estudiante como gestor de su propio conocimiento y aprendizaje, y ubica al docente como mediador, generando un espacio activo dentro de su clase, absuelve dudas e interrogantes, y en condiciones de virtualidad complementa la parte práctica del laboratorio físico con el uso de simuladores (Ribeiro et al., 2019).

El presente estudio realizado estuvo conformado por un grupo reducido de estudiantes, debido a que nuestra universidad es joven, y en los ciclos intermedios- superiores de la carrera profesional de ingeniería civil existen grupos pequeños de estudiantes matriculados (Albiter et al., 2019), lo que constituye una limitante para poder generalizar nuestros resultados, sin embargo muchas recomendaciones que se desprenden de esta indagación pueden ser aplicadas de forma pertinente en otras instituciones y realidades similares (Armstrong et al., 2020).

Por otro lado, la falta de recursos informáticos en una clase virtual no debe depender solamente de grandes inversiones generadas por nuestra institución educativa, como el montar grandes simuladores y sofisticados software como apoyo a la labor docente (Stahre et al., 2019), sino incorporar la necesidad de búsqueda y exploración por parte del docente. Cabe recalcar que existe un desfase generacional entre la gran mayoría de los estudiantes y docente en el uso de una aplicación informática para el desarrollo de una sesión de clase en física (Kurniawan et al., 2019), de tal forma que el docente muestre el uso de este software, así como incentive en su clase al uso de forma individual y colaborativa del mismo, generando un clima propicio para el aprendizaje y juego (Madden et al., 2020), así como una apertura a ideas nuevas para implementarlas, sobre todo en el contexto de la educación no presencial (Becerra et al., 2020).

Se evidencia dificultades en el desarrollo completo de las estructuras curriculares de física en la educación básica regular, es decir cuando el estudiante llega a la universidad, hay un desconocimiento de los tópicos de física (Estela et al., 2020), y por ende muy poco dominio 
de los conceptos avanzados de esta, de tal manera que la mayoría de ellos tienen dificultad en explicar el comportamiento dual de la luz o diferenciar la óptica física de la geométrica (Gonzáles et al., 2020).

Conforme a lo desarrollado en esta investigación, y al realizar nuestros respectivos cálculos para determinar el factor de Hake respecto a la ganancia en el aprendizaje conceptual de la óptica física y geométrica, se obtuvo valores de 0.65 y 0.60 que según la estandarización realizada por Richard Hake, corresponde a un nivel de ganancia media. En conclusión, se puede indicar que se evidenció un avance notorio relacionado al dominio conceptual enriquecido esta ganancia por las intervenciones realizadas por los estudiantes que nos colocan en situaciones nuevas (Martínez \& Riveros, 2019).

El usar aplicaciones informáticas en nuestras sesiones de clase no debe ser el fin, es decir no es el software, sino el cómo lo usemos. Es labor del docente dosificar el uso de aplicaciones en función al conocimiento pedagógico, manejo de recursos didácticos (Bustos, 2019), pero también del conocimiento del grupo de trabajo, ya que hay temas de física para los estudiantes que les cuesta más relacionarlo con su cotidianeidad, donde todo lo contrario, suelen indicar que requiere mayor grado de "abstracción", aunque esto último corresponde a la matemática que está detrás del fenómeno físicos. Es decir, lo que el docente tiene que ponderar en el uso de las tecnologías de información es el nivel de interactividad que puede generar entre estudiantes y con el docente, de esta forma se genera un ambiente virtual de juego y aprendizaje (Serrano, 2020).

Actualmente nuestra educación superior universitaria se desarrolla por competencias (Candela \& Restrepo, 2020), motivo por el cual el estudiante debe entre otras acciones, evidenciar su aprendizaje temático y dominio conceptual (Bezares et al., 2020) Por lo tanto, es necesario identificar la capacidad que tiene el estudiante para argumentar y exponer sus conjeturas respecto a una situación problemática planteada, acción que con la presente estrategia se evidencia de manera limitada en los momentos sincrónicos de la sesión de clase (Valderrama, 2021). Esto resulta una limitante de nuestra investigación, por lo tanto se sugiere en posteriores trabajos se evalúe utilizar otros tipos de herramientas que ofrecen las plataformas LMS como Moodle (Picón, 2020), para implementar una evaluación más integral y que permitan cristalizar las habilidades del estudiante en situaciones nuevas (Cornide \& Villarroel, 2019). 


\section{Conclusiones}

Esta investigación permite dejar en evidencia que el uso de simuladores en línea, como estrategia de enseñanza-aprendizaje en entornos virtuales, permite un mejor y mayor aprendizaje conceptual del tema de óptica geométrica en estudiantes universitarios. La ganancia normalizada obtenida en la temática de óptica geométrica fue de 0.65 y de óptica física 0.60 quedando demostrado que en nuestro grupo de estudiantes evolucionó favorablemente las concepciones físicas de esta temática, y que en función a las intervenciones en clase pueden extrapolar este conocimiento a situaciones nuevas cotidianas. En la actualidad las clases no presenciales permiten una oportunidad de aprendizaje mutuo para estudiantes-docentes, donde la brecha generacional se reduce en la medida que los docentes muestren apertura a las nuevas tecnologías y fomenten la interacción con los docentes para dar lugar a prácticas donde se desarrolle el trabajo autónomo y colaborativo.

\section{Referencias}

Albiter, J., Mendoza, R., \& Dorantes, E. (2019). El pensamiento computacional en la electrónica: la importancia del software de simulación en la comprensión del principio de funcionamiento de los componentes electrónicos. 3C TIC: Cuadernos de Desarrollo Aplicados a Las TIC, 8 (4), 85-113. https://doi.org/10.17993/3ctic.2019.84.85-113

Armstrong, E., Ramsey, K., Yankey, B., \& Self, S. (2020). COVID-19 and Distance Learning: Effects on Georgia State University School of Public Health Students. Frontiers in Public Health, 8 (September), 1-10. https://doi.org/10.3389/fpubh.2020.576227

Baidal, E., Cevallos, C., \& Bravo, F. (2019). Análisis del efecto de la aplicación de la Metodología de la Evaluación Formativa en la enseñanza de la Primera Ley de la Termodinámica. Latin American Journal of Science Education, 12058 (6), 1-18.

Barragán, F. (2020). Revista Ciencias de la Educación contextualizado de la Física universitaria. Revista Ciencias de La Educación, 30 (56), 541-568.

Becerra, D., Vargas, A., Boude, O., \& Benitez, M. (2020). Estrategias que apoyan el aprendizaje de la caída libre de los cuerpos. Espacios, 41 (48), 148-160. https://doi.org/10.48082/espacios-a20v41n48p11

Bezares, F., Toledo, G., Aguilar, F., \& Martínez, E. (2020). Aplicación de realidad aumentada centrada en el niño como recurso en un ambiente virtual de aprendizaje. Revista Científica Apertura, 12 (1), 88-105. https://doi.org/10.32870/Ap.v12n1.1820

Bustos, L. (2019). Diseño , implementación y análisis de una secuencia de enseñanza- 
aprendizaje acerca de cambio climático en el marco de la enseñanza para la comprensión para estudiantes de séptimo básico. Revista Innovación En Enseñanza de Las Ciencias, 3 (2), 65-99. https://doi.org/10.5027/reinnec.V3.I2.63

Cabrejos, S., Guerrero, E., \& Díaz, R. (2021). Experiencias en el uso del internet en estudiantes de enfermería de una universidad privada. ACC CIETNA: Revista de La Escuela de Enfermería, 8 (1), 45-53. https://doi.org/10.35383/cietna.v8i1.359

Candela, B., \& Restrepo, L. (2020). Enseñanza de la discontinuidad de la materia a través de la estrategia de Aprendizaje Basado en Problemas. Praxis, 16 (2), 199-214. https://doi.org/10.21676/23897856.3451

Castiblanco, O., Parmenio, X., León, Y., \& Vizcaíno, D. (2019). Enseñando a construir modelos explicativos de física en torno a juguetes. Infancias Imágenes, 18 (1), 21-35. https://doi.org/10.14483/16579089.12876

Castillo, N., Giraldo, D., \& Zapata, A. (2020). Aprendizaje por Descubrimiento: Método Alternativo en la Enseñanza de la Física. Scientia Et Technica, 25 (04), 569-575. https://doi.org/https://doi.org/10.22517/23447214.24221

Cifuentes, J. (2020). Docencia online y Covid-19: la necesidad de reinventarse. Revista de Estilos de Aprendizaje, 13 (3), 115-127.

Cornide, H., \& Villarroel, R. (2019). Método para Promover el Aprendizaje Colaborativo en Ingeniería de Software. Formación Universitaria, 12 (4), 3-12. https://doi.org/10.4067/s0718-50062019000400003

Cruz, M. (2020). Propuesta metodológica para el empleo del software educativo como recurso didáctico en la formación investigativa. Revista Ciencias Pedagógicas e Innovación, 7 (2), 21-30. https://doi.org/10.26423/rcpi.v7i2.288

Culzoni, C., Lescano, A., Demichelis, N., \& Bircher, G. (2020). Propuesta didáctica para la enseñanza de electromagnetismo basada en competencias. Revista de Enseñanza Para La Física, 32 (2), 7-18.

Culzoni, C., Panigatti, C., \& Bergesse, C. (2021). El proceso de enseñanza y aprendizaje en asignaturas de matemática para ingeniería durante el aislamiento por Covid 19. Revista Iberoamericana de Tecnología En Educación y Educación En Tecnología, 28, 70-82. https://doi.org/10.24215/18509959.28.e9

Delgado, N., Kiausowa, M., \& Escobar, A. (2021). Simulador virtual PhET para aprender Química en época de COVID-19. Journal of Chemical Information and Modeling, 53 $(9), 6$.

Espinoza, F. (2020). Impact of Guided Inquiry with Simulations on Knowledge of Electricity 
and Wave Phenomena. European Scientific Journal ESJ, 16 (33), 1-35. https://doi.org/10.19044/esj.2020.v16n33p1

Estela, R., \& Contreras, E. (2020). Propuestas interculturales para la enseñanza universitaria de Física basado en el saber ancestral awajún. Dekamu Agropec, 1 (2), 9-15.

Estela, R., Contreras, E., \& Incio, F. (2020). El juego Awajún como estrategia intercultural en la enseñanza de la física universitaria durante la educación no presencial. Revista Científica Epistemia, 4 (3), 152-165. https://doi.org/10.26495/re.v4i3.1428

Estela, R., Contreras, E., Rios, C., Cerna, H., Santamaría, N., Salcedo, A., \& Salcedo, D. (2021). Función intercultural de la historieta en la enseñanza de la física y matemática universitaria. South Florida Journal of Development, 2 (1), 395-404. https://doi.org/10.46932/sfjdv2n1-029

Flores, F., Gallegos, L., \& Lima, C. (2020). Representaciones en Física: construcción y validación de un cuestionario para la enseñanza superior. Revista Electronica de Investigacion Educativa, $22 \quad$ (1), 1-17. https://doi.org/10.24320/REDIE.2020.22.E14.1728

García, V., \& Silva, M. (2019). Procrastinación académica entre estudiantes de cursos en línea. Validación de un cuestionario. Revista Científica Apertura, 11 (2), 122-137. https://doi.org/10.32870/ap.v11n2.1673

Gonzáles, E., Muñoz, Z., \& Solbes, J. (2020). Enseñanza de la Física: Una comparativa de tres países. Góndonla, Enseñanza y Aprendizaje de Las Ciencias, 15 (2), 239-250. https://doi.org/10.14483/23464712.15619

Gutiérrez, R., \& Castillo, L. (2020). Simulators with Geogebra Software as Learning Objects For Teaching Physics. Tecné Episteme y Didaxis: TED, 47, 201-216. https://doi.org/10.17227/ted.num47-11336

Jimenez, F., Beleño, L., Agudelo, J., \& Muñiz, J. (2020). Unidades didácticas en física como potenciadores del aprendizaje en estudiantes de ingeniería. Formación Universitaria, 13 (6), 143-154. https://doi.org/10.4067/s0718-50062020000600143

Kurniawan, R., Mujasam, M., Yusuf, I., \& Widyaningsih, S. (2019). Development of physics learning media based on Lectora Inspire Software on the elasticity and Hooke's law material in senior high school. Journal of Physics: Conference Series, 1157 (3), 1-8. https://doi.org/10.1088/1742-6596/1157/3/032022

Lara, E., Rebolledo, G., \& Rojano, J. (2019). Mejorando el aprovechamiento de las actividades colaborativas por pares de estudiantes utilizando tecnología educativa en matemática. Revista Complutense de Educación, $30 \quad$ (2), 441-460. 
https://doi.org/10.5209/rced.57597

Madden, J., Pandita, S., Schuldt, J., Kim, B., Won, A. S., \& Holmes, N. G. (2020). Ready student one: Exploring the predictors of student learning in virtual reality. Plos One, 15 (3), 1-26. https://doi.org/10.1371/journal.pone.0229788

Martínez, J., Guilcapi, J., López, J., \& Mata, M. (2020). El laboratorio virtual mediante el simulador Modellus 4.01 y su incidencia en el aprendizaje de la dinámica traslacional. Polo de Conocimiento, 5 (06), 82-98. https://doi.org/DOI: 10.23857/pc.v5i6.1468

Martínez, N., \& Riveros, S. (2019). La enseñanza de caída libre bajo la metodología de aprendizaje activo. Tecné Episteme y Didaxis: TED, 45 (1), 35-56. https://doi.org/10.17227/ted.num45-9832

Medina, J., Rampirez, M., \& Miranda, I. (2019). Validez y confiabilidad de un test en línea sobre los fenómenos de reflexión y refracción del sonido. Revista Científica Apertura, 11 (2), 104-121. https://doi.org/10.32870/Ap.v11n2.1622

Merino, C., Moreira, P., \& Marzabal, A. (2019). Análisis sistémico de la evolución de los componentes del modelo eléctrico de los estudiantes. Didacticae, 5, 26-42. https://doi.org/10.1344/did.2019.5.26-42

Miranda, C., \& Romero, R. (2019). Un software educativo como una herramienta pedagógica en la mejora de las habilidades de lectoescritura utilizando el método ecléctico An educational software as a pedagogical tool in the improvement of reading and writ. Tecnología, Ciencia y Educación, 13, 172-186. https://doi.org/10.51302/tce.2019.291

Naizaque, N. (2020). Implementación de CPBL en física mecánica para el desarrollo de habilidades de orden superior en estudiantes de ingeniería. Latin-American Journal of Physics Education, 14 (3), 1-7.

Perdomo, G., \& Galo, A. (2020). Aula invertida como estrategia didáctica para propiciar el aprendizaje conceptual de la Mecánica de Fluidos de Física General en el nivel superior. Revista de La Escuela de Física, 8 (1), 44-59. https://doi.org/10.5377/ref.v8i1.10088

Picón, M. L. (2020). ¿Es posible la enseñanza virtual? Foro educacional, 34, 11-34. DOI: https://doi.org/10.29344/07180772.34.2357

Ribeiro, A., Dionizio, M., Bovolenta, D., Oliveira, R., \& Colombo, P. (2019). Prácticas innovadoras en la enseñanza de física moderna en una escuela pública brasileña. Revista de Estudios y Experiencias En Educación, 18 (36), 241-256. https://doi.org/10.21703/rexe.20191836ribeiro2

Rodríguez, J., Ayala, G., \& López, M. (2019). Aprovechamiento escolar en aritmética: Objeto de aprendizaje en lengua de señas mexicana para sordos. Revista Iberoamericana Para 
La Investigación y El Desarrollo Educativo, $10 \quad$ (19), 1-28. https://doi.org/10.23913/ride.v10i19.558

Rondon, M., Caballero, R., Baleta, L., \& García, T. (2020). La modelación matemática y sus aportes en la formación del licenciado. Espacios, 41 (30), 72-84. https://www.revistaespacios.com/a20v41n14/in204130.html

Serrano, R. (2020). Aplicación de las TIC en la enseñanza del electromagnetismo para carreras de ingeniería. Informática y Sistemas, 4 (1), 1-6.

Siswoyo, S., \& Muliyati, D. (2021). Teaching high school physics using PhET interactive simulation. AIP Conference Proceedings, 2331 (abril), 0-7. https://doi.org/10.1063/5.0041657

Solbes, J., Muñoz, Z., \& Ramos, G. (2019). Enseñanza de la estructura atómica de la materia en Colombia. Revista Historia y Educación, 22 (22), 117-140.

Solé, A., Aguilar, D., \& Ibañez, M. (2020). The teacher role in school inquiries through simulation tools. Revista Electrónica de Tecnología Educativa, 74, 221-233. https://doi.org/10.21556/edutec.2020.74.1803

Stahre, B., Eriksson, T., Karlsson, G., Sunnerstam, M., Axelsson, M., \& Billger, M. (2019). Design considerations for virtual laboratories: A comparative study of two virtual laboratories for learning about gas solubility and colour appearance. Education and Information Technologies, 24 (3), 2059-2080. https://doi.org/10.1007/s10639-01809857-0

Torres, C., Vargas, J., \& Cuero, J. (2020). Modelo didáctico para la enseñanza - aprendizaje de la física mecánica a nivel universitario. Espacios, 41 (20), 22-36. https://doi.org/10.5281/zenodo.4310285

Valderrama, D. (2021). Competencias matemáticas: una mirada desde las estrategias de enseñanza en educación a distancia. Góndola, Enseñanza y Aprendizaje de Las Ciencias, 16 (2), 382-398. https://doi.org/10.14483/23464712.16167

Zenteno, F., Carhuachín, A., \& Rivera, T. (2020). Uso de software educativo interactivo para la enseñanza y aprendizaje de la matemática en educación básica, Región Pasco. $\begin{array}{lllll}\text { Horizonte } & \text { de } & \text { La } & \text { Ciencia, } & 10\end{array}$ https://doi.org/10.26490/uncp.horizonteciencia.2020.19.596 